\title{
Influences of the Filter Effect on Pulse Splitting in Passively Mode-Locked Fiber Laser with Positive Dispersion Cavity
}

\author{
Xiaodong Chen* \\ State Key Laboratory of Pulsed Power Laser Technology, Electronic Engineering Institute, \\ Hefei 230037, China
}

(Received October 14, 2014 : revised January 26, 2015 : accepted February 23, 2015)

\begin{abstract}
Based on the extended nonlinear Schrödinger equation, the influences of the filter effect on pulse splitting in a passively mode-locked erbium-doped fiber laser with positive dispersion cavity are investigated theoretically. Numerical results show that, as the bandwidth of the spectral filter decreases, the nonlinear chirp appended to the pulse increases under the combined action of the filter effect of the super-Gaussian spectral filter and the self-phase modulation effect. On further decreasing the bandwidth, the wave breaking of the pulse takes place. In addition, by varying the pump power of the laser or the profile of the spectral filter, the influences of the filter effect on pulse splitting also change accordingly.
\end{abstract}

Keywords : Fiber laser, Passively mode-locked, Dissipative soliton, Spectral filter

OCIS codes : (060.3510) Lasers, fiber; (140.4050) Mode-locked lasers; (190.5530) Pulse propagation and solitons

\section{INTRODUCTION}

High-repetition-rate and large energy ultra-short laser pulses are very important for applications of nonlinear frequency conversion, ultrafast dynamics exploration, and optical precision procession [1-3]. Rare-earth doped silica-based fibers are very good gain media for building mode-locked lasers generating such ultra-short pulses [4-8]. However, the nonlinear Kerr effect in the long-range transmission fibers induced by the ultra-short pulses with high peak power is very serious. This may result in a very high nonlinear phase-shifting. When the dispersion cannot control or compensate the accumulated nonlinear phase-shifting, the nonlinear effect may induce nonlinear chirps to cause a pulse distortion [9], which makes it difficult to obtain large energy pulses. Previous research shows that a fiber laser with all-normal group-velocity dispersion (GVD) or large normal GVD together with small anomalous GVD can achieve a dissipative soliton (DS) with large energy in the mode-locked pulse shaping [10,11]. The formation mechanism of DSs is attributed to an integrated result of all kinds of effects in the laser cavity, such as the spectral filter effect of the gain medium, Kerr nonlinear effect, normal dispersion, nonlinear polarization rotation (NPR) or saturable absorber (SA), gain and loss of the laser cavity, etc. It has also been found that a spectral filter (SF) inserted in a passively mode-locked fiber laser with a positive dispersion cavity can be used to balance the pulse chirp for achieving stable DSs. Moreover, the pulse shape (pulse duration, peak power, etc.) and the spectrum (spectral width, etc.) of the DS are closely related to the bandwidth of the SF [11-14]. In fact, the variation of the SF bandwidth would not only result in changes of the pulse shape and spectrum of the DS, but would also induce the pulse to cause the wave breaking, which affects the stabilization of the output pulse and the increment of the pulse energy. Therefore, the influences of the filter effect on pulse splitting in a passively mode-locked fiber laser with positive dispersion cavity are worth in-depth study.

In this paper, the influences of the filter effect on pulse splitting in a passively mode-locked erbium-doped fiber laser (EDFL) with positive dispersion cavity are investigated theoretically. Our results show that as the SF bandwidth decreases, the nonlinear chirp appended to the pulse increases under the combined action of the filter effect of the superGaussian SF and the self-phase modulation (SPM) effect. On further decreasing the bandwidth, the wave breaking of the pulse takes place. In addition, by varying the pump power of the laser or the profile of the SF, the influences

*Corresponding author: chenxd503@hotmail.com 
of the filter effect on pulse splitting would change accordingly.

\section{THEORETICAL MODEL}

Figure 1 shows the propagation model of a passively modelocked EDFL with an SF. The pump light is launched into the EDF through a wavelength division multiplexing (WDM) coupler. The positive dispersion ring cavity is comprised of a single-mode fiber (SMF) and the EDF. The polarization additive-pulse mode-locked (PAPM) system is made of a polarization-sensitive isolator and two sets of polarization controllers. The PAPM is used to produce the NPR effect. An SF inserted in the cavity after the PAPM has a variable bandwidth. The $10 \%$ port of a 10:90 optical coupler (OC) is used for the laser pulse output.

By solving the extended nonlinear Schrödinger equation (NLSE), the operation of a passively mode-locked fiber laser with a positive dispersion cavity can be numerically simulated. The equation is given by $[15,16]$

$$
\frac{\partial A}{\partial z}+i \frac{\beta_{2}}{2} \frac{\partial^{2} A}{\partial t^{2}}=g A+i \gamma|A|^{2} A+\frac{g}{2 \Omega_{g}^{2}} \frac{\partial^{2} A}{\partial t^{2}},
$$

where $A$ is the pulse envelope amplitude, $\beta_{2}$ represents the fiber dispersion, $\gamma$ is the nonlinearity parameter, $\Omega_{g}$ is the bandwidth of the laser gain. The gain function for the EDF is described by $[17,18]$

$$
g=g_{0} \exp \left(-\frac{E_{p}}{E_{s}}\right)
$$

Here $g_{0}$ is the small signal gain coefficient. $E_{s}$ is the gain saturation energy, which is related to the laser pump power. The pulse energy is given by

$$
E_{p}=\int_{-T_{R} / 2}^{T_{R} / 2}|A(z, \zeta)|^{2} d \zeta
$$

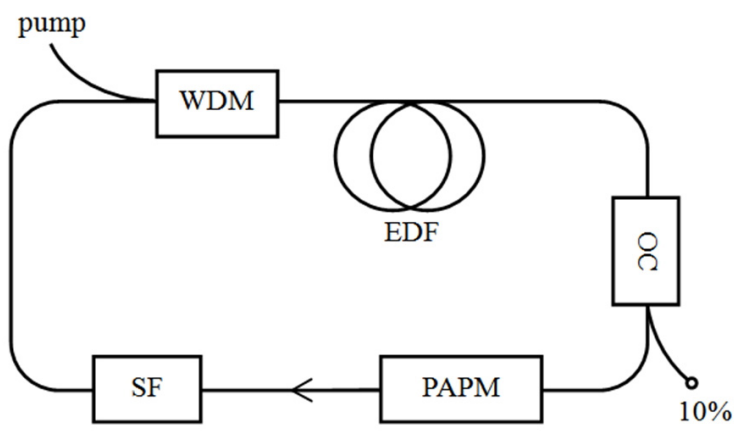

FIG. 1. Illustration of the fiber laser cavity elements used for the proposed model. EDF, erbium-doped fiber; WDM, wavelength division multiplexing; PAPM, polarization additive-pulse mode-locked; OC, optical coupler; SF, spectral filter. where $T_{R}$ is the cavity round-trip time.

The gain medium of the EDF has a gain bandwidth of $25 \mathrm{~nm}$, with profile of super-Gaussian type. The PAPM element is a similar SA [11], which can serve as the spectral and temporal filtering element $[11,12,19,20]$. The PAPM element has a super-Gaussian transmission function with the bandwidth of $70 \mathrm{~nm}$.

A scalar model to simplify the propagation model with the PAPM function, so the intensity-depended transmission function of the PAPM element is given by [7]

$$
T=0.94 \times\left(I(t) / I_{\max }\right)^{2},
$$

where $I(t)$ is the pulse intensity distribution of the time $t$, and $I_{\max }$ is the maximum value of $I(t)$. An SF inserted in the cavity has a super-Gaussian profile, and the filtering function is given by

$$
T_{S F}=\exp \left[-\left(\frac{\omega}{\Omega_{S F}}\right)^{4}\right]
$$

where $\omega$ is the angular frequency, $\Omega_{S F}$ is the bandwidth of SF. In fact, SF could also be seen as an effective SA, which cuts off the temporal wings of a pulse.

The total length of laser cavity is $14.5 \mathrm{~m}$ with the net cavity dispersion of $\sim 0.44 \mathrm{ps}^{2}$. A pulse with Gaussian shape is used as an initial condition, and the initial chirp parameter $\mathrm{C}$ is zero. In accordance with Eq. (1), by using the standard split step Fourier method, the laser operation in a passively mode-locked fiber laser can be simulated with the parameters displayed in Table 1. In the simulation, we use the shooting method to determine whether the laser reaches the steady state. Based on the self-reproduction features of the laser, only when the difference between the initial value and the calculated value is within given precision limitation do we believe that the laser reaches the steady state.

\section{RESULTS AND DISCUSSIONS}

Initially, the influences of the filter bandwidth on pulse output properties are investigated. In the simulation, the gain saturation energy $E_{s}$ is fixed at $0.3 \mathrm{~nJ}$. Figure 2 shows

TABLE 1. Fiber parameters used in the simulation of the laser cavity

\begin{tabular}{c|c|c|c}
\hline \hline Parameter & EDF & SMF & Units \\
\hline Length & 10 & 4.5 & $\mathrm{~m}$ \\
\hline Dispersion $\left(\beta_{2} @ 1550 \mathrm{~nm}\right)$ & $+53.5 \times 10^{-3}$ & $-21.7 \times 10^{-3}$ & $\mathrm{ps}^{2} / \mathrm{m}$ \\
\hline Nonlinear coefficient $(\gamma)$ & 4.5 & 1.3 & $\mathrm{~W}^{-1} \mathrm{~km}^{-1}$ \\
\hline Gain bandwidth $\left(\Delta \lambda_{g}\right)$ & 25 & & $\mathrm{~nm}$ \\
\hline Small signal gain $\left(g_{0}\right)$ & 2 & & $\mathrm{~m}^{-1}$ \\
\hline
\end{tabular}




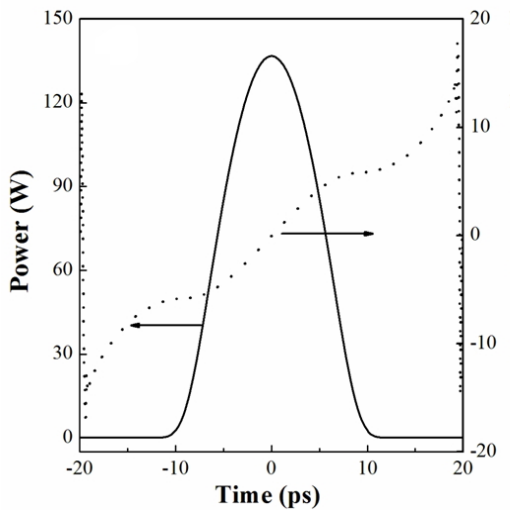

(a)

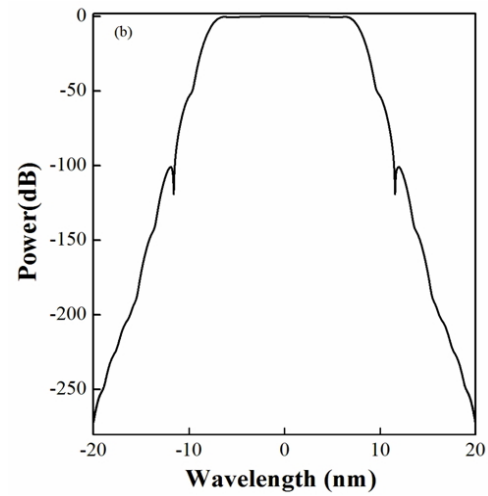

(b)

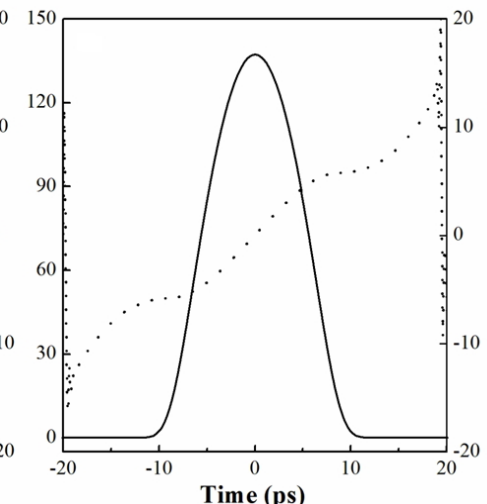

(c)

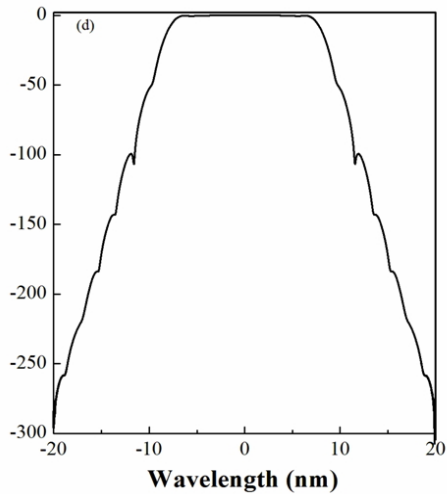

(d)

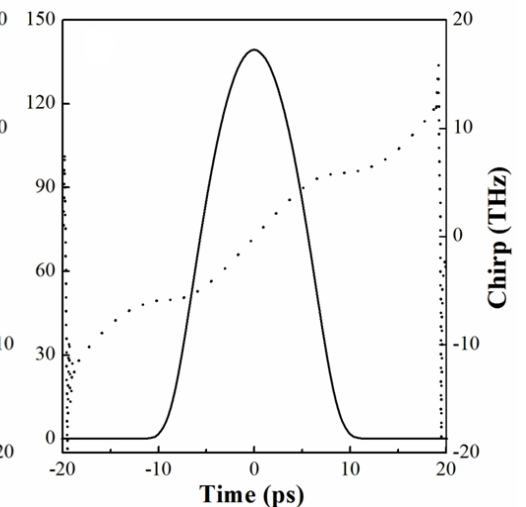

(e)

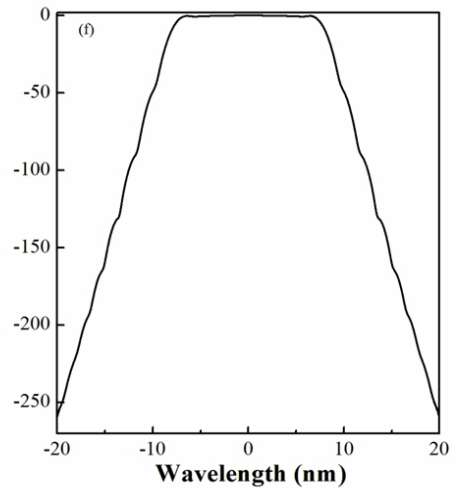

(f)

FIG. 2. Temporal power profile (solid curve) and chirp (dashed curve) and spectral power profile at the output position for the different bandwidths of the SF. (a)-(b): $50 \mathrm{~nm}$, (c)-(d): $35 \mathrm{~nm}$, (e)-(f): $25 \mathrm{~nm}$.

the simulation results for different SF bandwidths. As shown, the output pulse spectrum has a flat top with steep edges and is similar to the trapezoid-spectrum shape. The pulse duration and peak power can reach several picoseconds and above 100 watts, respectively. This means that the large energy DSs have been achieved in a passively modelocked fiber laser with a positive dispersion cavity. When the SF bandwidths are 50, 35 and $25 \mathrm{~nm}$, the pulse durations are $11.64,11.6$ and $11.4 \mathrm{ps,}$ the peak powers are 136.7, 137.2 and $139.3 \mathrm{~W}$, and the spectral widths are 17.54, 17.6 and $17.76 \mathrm{~nm}$, respectively. One can clearly see that the pulse shapes and the spectra vary slowly with decreasing SF bandwidth. The pulse duration is decreasing and the peak power becomes higher, and the pulse spectrum broadens accordingly. The reason for this may be explained as follows. When the SF bandwidth exceeds the pulse spectral width, the filter effect is very weak. Only the leading and trailing edges of the pulse spectral envelope can be filtered, which makes the central part of the pulse obtain higher gain than the edges. From the maps of the temporal evolution of the pulse width and spectral bandwidth in one round trip [7, 11], there is an abrupt change for the pulse temporal waveforms and the spectra. This corresponds to narrowing down the widths of both the pulse shape and the spectrum as the pulse passes through the filter. The narrower the bandwidth of the SF, the bigger the changes. When the pulse gets a balance, a stable pulse can be achieved with the broader spectrum, the narrower pulse shape and the higher peak power.

It can also be seen that the pulse chirp doesn't satisfy the linear evolution, but the accumulation of the nonlinear chirp is not enough to make the pulse split as the SF bandwidth decreases. The reason is that the filter has less impact on the pulse for the broader SF, and the pulse peak power increase is limited for the decrease of the SF bandwidth. Given that the nonlinear chirp is caused by the combination of the filter effect and the SPM effect, the impact on the nonlinear chirp accumulation of the pulse is limited.

Figure 3 shows the output pulse shape, chirp and spectrum with a decrease to $15 \mathrm{~nm}$ of the SF bandwidth. As shown, the output pulse spectral width increases to $21.04 \mathrm{~nm}$ rapidly. Meanwhile, the output pulse shape narrows down to $10.4 \mathrm{ps}$, and the peak power rapidly increases to 157.1 W. At this point, the SF bandwidth is less than the pulse spectral width. When the pulse passes the filter, not only does the pulse shape become narrower, but the spectral wings can be cut off, so both the pulse shape and the spectrum have obvious changes. It also indicates that the nonlinear chirp increases along with the decrease of the SF bandwidth. This is because the filter effect begins to be notable when the SF bandwidth can compare to the pulse 


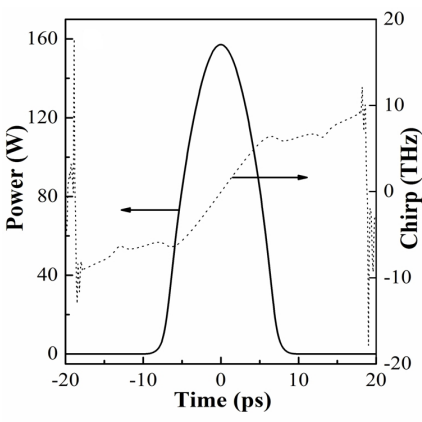

(a)

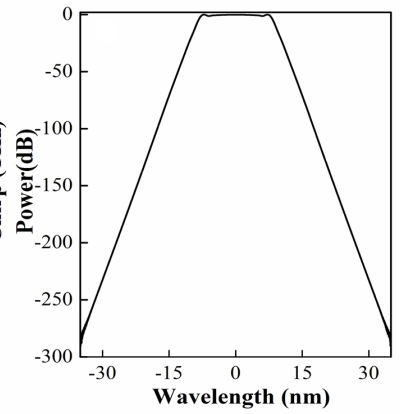

(b)
FIG. 3. (a) Temporal power profile (solid curve) and chirp (dashed curve) and (b) spectral power profile at the output position with the SF bandwidth of $15 \mathrm{~nm}$.

spectral width. Meanwhile, the influences of the SPM effect are enhanced greatly for the rapid increase of the pulse peak power, which results in output pulse containing more nonlinear chirp. With the action of the combination of the filter effect and the SPM effect, the pulse chirp deviates seriously from the linear distribution. It is worthwhile to point out that, as the nonlinear chirp increases, the wave breaking is more likely to occur, so it can be seen that the pulse would split if the SF bandwidth further decreases.

Figure 4(a)-(b) shows the output pulse shape, chirp and spectrum with the SF bandwidth of $9 \mathrm{~nm}$. Here we can see that the output pulse spectrum further broadens and begins to deviate from the trapeziform distribution. Meanwhile, the pulse shape obviously changes and at the edges small fluctuations emerge. By comparing the results shown in Fig. 4(c)-(d) with the SF bandwidth of $7 \mathrm{~nm}$ to those in Fig. 4(a)-(b), we clearly see that the small fluctuations have evolved into multi-peak oscillation structures. This indicates that the pulse is beginning to split. The nonlinear chirp increases quickly with the further decrease of the SF bandwidth, so the different parts of the pulse spectral envelopes have the same instantaneous frequency, thus interfering with each other, which leads to the edges with multi-peak oscillation structures.

It is worth pointing out, as an SF is introduced in the laser cavity, it modifies the laser attracting state in some way [11]. When the SF band is wide, the round-trip cavity length is long enough so that after filter application the pulse can return to its attracting state before encountering the SF again. With the decreasing of SF bandwidth, the laser attracting state would not change. Finally we can see that the pulse duration decreases, while the spectral width and the pulse peak power increase, but both the pulse temporal waveforms and the spectra are similar. When the SF band is very narrow, SF has a strong filtering effect. The pulse cannot return to its original attracting state when the pulse encountering SF again, so the laser attracting state would change. Both the pulse temporal waveforms and the spectra have big changes, which could eventually lead to the wave breaking.

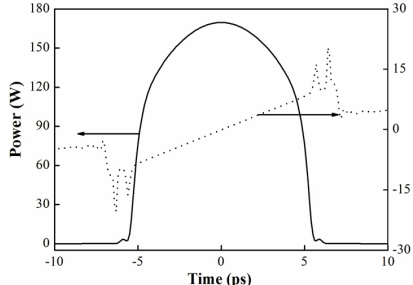

(a)

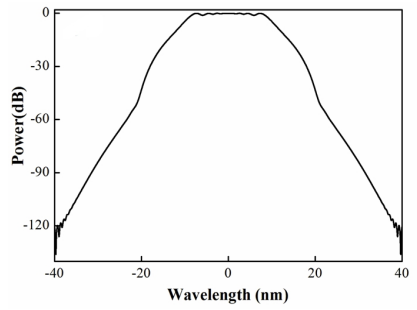

(b)

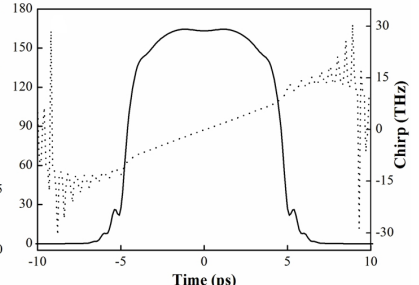

(c)

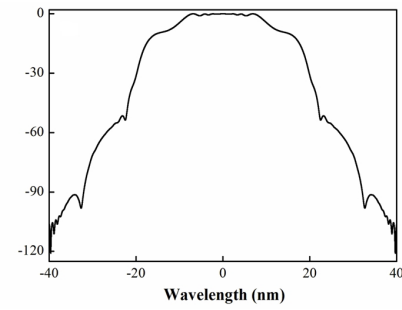

(d)
FIG. 4. Temporal power profile (solid curve) and chirp (dashed curve) and spectral power profile at the output position for the different bandwidth of the SF. (a)-(b): $9 \mathrm{~nm}$, (c)-(d): $7 \mathrm{~nm}$.

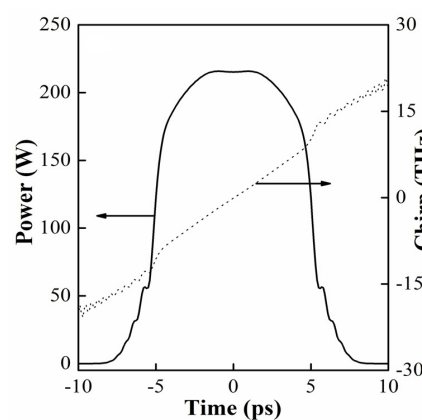

(a)

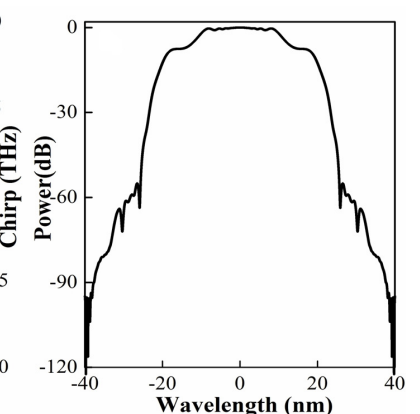

(c)
FIG. 5. (a) Temporal power profile (solid curve) and chirp (dashed curve) and (b) spectral power profile at the output position with the SF bandwidth of $7 \mathrm{~nm}$ when the gain saturation energy $E_{s}$ is $0.45 \mathrm{~nJ}$.

The above results show that the pulse splitting in a passively mode-locked fiber laser with positive dispersion cavity is influenced by the SF bandwidth when the laser pump power is constant. In fact, the pulse shape and spectrum are related to the laser pump power, the variation of the pump power can lead to the changes of the pulse splitting characteristics with a certain bandwidth SF.

Figure 5 shows the pulse shape, chirp and spectrum when $E_{S}$ is $0.45 \mathrm{~nJ}$ and the SF bandwidth is $7 \mathrm{~nm}$. Compared to the results in Fig. 4(c)-(d), the pulse is broadened in both time and frequency domains, and the peak power is also increased. Meanwhile, the multi-peak oscillation structures of the pulse edges become more obvious. As the laser pump power is increased, the phenomenon of wave breaking is clearer. The reason is that both the pulse duration and spectral width increase with the pump power, so the filter 


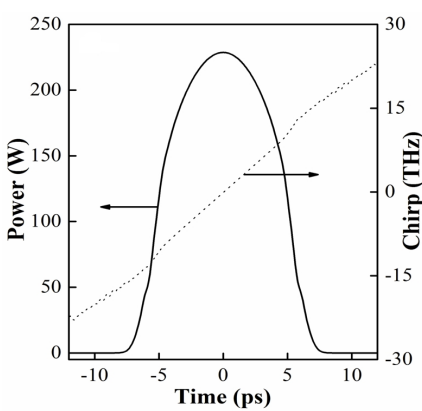

(a)

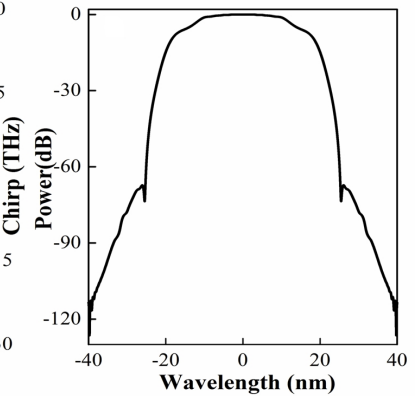

(c)
FIG. 6. (a) Temporal power profile (solid curve) and chirp (dashed curve) and (b) spectral power profile at the output position with the Gaussian SF bandwidth of $7 \mathrm{~nm}$ when the gain saturation energy $E_{s}$ is $0.45 \mathrm{~nJ}$.

effect of the SF is strengthening for the same SF bandwidth and the SPM effect is enhanced for the increase of the pulse peak power.

In addition, since the super-Gaussian SF brings in the nonlinear chirp, which can make the pulse split. In the case shown in Fig. 6, a Gaussian SF is used in the laser cavity instead while $E_{s}$ of $0.45 \mathrm{~nJ}$ and the SF bandwidth of $7 \mathrm{~nm}$ remain unchanged. As shown, the nonlinear chirp decreases and the multi-peak oscillation structures of the pulse edges are weakening to small fluctuations. Because the Gaussian SF does not induce the nonlinear chirp, the nonlinear chirp is only related to the SPM effect. The wave breaking effect may be weak with the decrease of the nonlinear chirp.

\section{CONCLUSION}

We have investigated theoretically the influences of the filter effect on pulse splitting in a passively mode-locked EDFL with a positive dispersion cavity based on the extended NLSE. Our results have shown that as the SF bandwidth decreases, the nonlinear chirp appended to the pulse increases under the combined action of the filter effect of the super-Gaussian SF and the SPM effect. On further decreasing the bandwidth, the wave breaking of the pulses takes place. In addition, by varying the pump power of the laser or the profile of SF, the influences of the filter effect on pulse splitting also change accordingly. As the laser pump power is increased, the phenomenon of wave breaking is clearer. By using a Gaussian SF in the laser cavity instead, the wave breaking effect may be weak with the decrease of the nonlinear chirp.

\section{REFERENCES}

1. C. B. Schaffer, A. Brodeur, J. F. García, and E. Mazur, "Micromachining bulk glass by use of femtosecond laser pulses with nanojoule energy," Opt. Lett. 26, 93-95 (2001).
2. Y. M. Wang, H. Shen, L. Q. Hua, C. J. Hu, and B. Zhang, "Predissociation dynamics of the B state of $\mathrm{CH}_{3} \mathrm{I}$ by femtosecond pump-probe technique," Opt. Express 17, 10506-10513 (2009).

3. F. Korte, S. Adams, A. Egbert, C. Fallnich, A. Ostendorf, S. Nolte, M. Will, J. P. Ruske, B. Chichkov, and A. Tuennermann, "Sub-diffraction limited structuring of solid targets with femtosecond laser pulses," Opt. Express 7, 41-49 (2000).

4. A. Ruehl, D. Wandt, U. Morgner, and D. Kracht, "Normal dispersive ultrafast fiber oscillators," IEEE J. Select. Topics Quantum Electron. 15, 170-181 (2009).

5. H. Zhang, D. Y. Tang, L. M. Zhao, X. Wu, and H. Y. Tam, "Dissipative vector solitons in a dispersion- managed cavity fiber laser with net positive cavity dispersion," Opt. Express 17, 455-460 (2009).

6. A. Haboucha, A. Komarov, H. Leblond, F. Sanchez, and G. Martel, "Mechanism of multiple pulse formation in the normal dispersion regime of passively mode-locked fiber ring lasers," Opt. Fiber Technol. 14, 262-267 (2008).

7. X. M. Liu, "Numerical and experimental investigation of dissipative solitons in passively mode-locked fiber lasers with large net-normal-dispersion and high nonlinearity," Opt. Express 17, 22401-22416 (2009).

8. L. A. Vazquez-Zuniga and Y. C. Jeong, "Wavelength-tunable, passively mode-locked erbium-doped fiber master-oscillator incorporating a semiconductor saturable absorber mirror," J. Opt. Soc. Korea 17, 117-129 (2013).

9. G. P. Agrawal, Nonlinear Fiber Optics (Academic Press, Boston, USA, 2007).

10. N. Akhmediev and A. Ankiewicz, Dissipative Solitons (Springer, Berlin, Germany, 2005).

11. F. W. Wise, A. Chong, and W. Renninger, "High-energy femtosecond fiber lasers based on pulse propagation at normal dispersion," Laser Photonics Rev. 2, 58-73 (2008).

12. B. G. Bale, J. N. Kutz, A. Chong, W. H. Renninger, and F. W. Wise, "Spectral filtering for high-energy mode-locking in normal dispersion fiber lasers," J. Opt. Soc. Am. B 25, 1763-1770 (2008).

13. X. H. Li, Y. S. Wang, W. Zhao, W. Zhang, Z. Yang, X. H. Hu, H. S. Wang, X. L. Wang, Y. N. Zhang, Y. K. Gong, C. Li, and D. Y. Shen, "All-normal dispersion, figure-eight, tunable passively mode-locked fiber laser with an invisible and changeable intracavity bandpass filter," Laser Phys. 21, 940-944 (2011).

13. L. A. Vazquez-Zuniga, Y. C. Jeong, "Power-scalable, subnanosecond mode-locked erbium-doped fiber laser based on a frequency-shifted-feedback ring cavity incorporating a narrow bandpass filter," J. Opt. Soc. Korea 17, 177-181 (2013).

14. A. Cabasse, B. Ortaç, G. Martel, A. Hideur, and J. Limpert, "Dissipative solitons in a passively mode-locked Er-doped fiber with strong normal dispersion," Opt. Express 16, 1932219329 (2008).

15. X. H. Li, Y. S. Wang, W. Zhao, X. L. Liu, Y. G. Wang, Y. H. Tsang, W. Zhang, X. H. Hu, Z. Yang, C. X. Gao, C. Li, and D. Y. Shen, "All-fiber dissipative solitons evolution in a compact passively $\mathrm{Yb}$-doped mode-locked fiber laser," J. Lightwave Technol. 30, 2502-2507 (2012).

16. L. M. Zhao, D. Y. Tang, H. Y. Tam, and C. Lu, "Pulse breaking recovery in fiber lasers," Opt. Express 16, 12102-12107 (2008). 
17. G. P. Agrawal, "Amplification of ultrashort solitons in erbium-doped fiber amplifiers," IEEE Photon. Technol. Lett. 2, 875-877 (1990).

18. A. Chong, J. Buckley, W. Renninger, and F. Wise, "All-normaldispersion femtosecond fiber laser," Opt. Express 14, 1009510100 (2006).
19. G. Martel, C. Chédot, A. Hideur, and P. Grelu, "Numerical maps for fiber lasers mode locked with nonlinear polarization evolution: Comparison with semi-analytical models," Fiber Integr. Opt. 27, 320-340 (2008). 